\title{
Optimal input design for fault detection and diagnosis
}

\section{Sadegh, Payman; Madsen, Henrik; Holst, J.}

\section{Published in:}

Proceedings of the American Control Conference

Publication date:

1995

\section{Document Version}

Publisher's PDF, also known as Version of record

Link back to DTU Orbit

Citation (APA):

Sadegh, P., Madsen, H., \& Holst, J. (1995). Optimal input design for fault detection and diagnosis. In Proceedings of the American Control Conference (Vol. Volume 2, pp. 1147-1148). IEEE.

\section{General rights}

Copyright and moral rights for the publications made accessible in the public portal are retained by the authors and/or other copyright owners and it is a condition of accessing publications that users recognise and abide by the legal requirements associated with these rights.

- Users may download and print one copy of any publication from the public portal for the purpose of private study or research.

- You may not further distribute the material or use it for any profit-making activity or commercial gain

- You may freely distribute the URL identifying the publication in the public portal

If you believe that this document breaches copyright please contact us providing details, and we will remove access to the work immediately and investigate your claim 


\title{
Optimal Input Design for Fault Detection and Diagnosis
}

\author{
P. SADEGH* ${ }^{*}$ H. MADSEN* ${ }^{*}$ and J. HOLST ${ }^{\dagger}$ \\ * Inst. of Mathematical Modeling, Technical University of Denmark, \\ DK-2800 Lyngby, Denmark. \\ $\dagger$ Dept. of Mathematical Statistics, Lund Institute of Technology, \\ S-22100 LUND, Sweden.
}

\begin{abstract}
In the paper, the design of optimal input signals for detection and diagnosis in a stochastic dynamical system is investigated. The design is based on maximization of Kullback measure between the model under fault and the model under normal operation conditions. It is established that the optimal input design for change detection when the magnitude of change is small is equivalent to optimal input design for parameter estimation.
\end{abstract}

\section{Kullback measure and input design}

Definition 1.1 The Kullback-Leiber information for discriminating between two models $\mathcal{M}_{0}$ and $\mathcal{M}_{1}$ is defined by

$$
K\left(\mathcal{M}_{1}, \mathcal{M}_{0}\right)=\int \log \frac{p_{1}(y)}{p_{0}(y)} p_{1}(y) d y
$$

where $p_{0}(y)$ and $p_{1}(y)$ are the probability densities of the data under $\mathcal{M}_{0}$ and $\mathcal{M}_{1}$ respectively.

Theorem 1.1 Assume that the two SISO models:

$$
\mathcal{M}_{0}: y(k \Delta T)=G_{1,0}(\delta) u(k \Delta T)+G_{2,0}(\delta) e(k \Delta T)
$$$$
\mathcal{M}_{1}: y(k \Delta T)=G_{1,1}(\delta) u(k \Delta T)+G_{2,1}(\delta) e(k \Delta T)
$$

are to be tested against each other where the $\delta$ operator is defined by (cf [1])

$$
\delta=\frac{q-1}{\Delta T}
$$

$q$ is the forward shift operator and $\Delta T$ is the sampling time. Also assume that

- $\{e(k \Delta T)\}$ is a sequence of uncorrelated zero mean Gaussian random variables.
- The input signal admits a spectral representation and is power restricted.

- The length of the experiment is large.

- There is no feed-back in the system.

Then maximizing the Kullback-Leiber measure $K\left(\mathcal{M}_{1}, \mathcal{M}_{0}\right)$ with respect to the power restricted input is equivalent to the optimization problem

$$
\begin{aligned}
& \max \int_{0}^{\frac{\pi}{\Delta T}}\left|\frac{\Delta G_{1}^{\prime}\left(e^{j \omega \Delta T}\right)}{G_{2,0}^{\prime}\left(e^{j \omega \Delta T}\right)}\right|^{2} d \xi(\omega) \\
& \int_{0}^{\frac{\pi}{\Delta T}} d \xi(\omega)=1
\end{aligned}
$$

where for each transfer function, we denote $G(\delta)=$ $G^{\prime}(q) . \xi(\omega)$ is the power distribution of the input defined over the range of frequencies $\left[0, \frac{\pi}{\Delta T}\right]$. The solution is given by

$$
\xi(\omega)= \begin{cases}0 & \omega<\omega^{*} \\ 1 & \omega \geq \omega^{*}\end{cases}
$$

where

$$
\omega^{*}=\arg \max _{\omega}\left|\frac{\Delta G_{1}\left(\frac{e^{j \omega \Delta T}-1}{\Delta T}\right)}{G_{2,0}\left(\frac{e^{j \omega \Delta T}-1}{\Delta T}\right)}\right|
$$

and

$$
\Delta G_{1}(\delta)=G_{1,1}(\delta)-G_{1,0}(\delta)
$$

Proof: The proof is based on direct computation of the Kullback measure. For details see [2].

Remark 1.1 If we denote the parameter in $G_{1,0}$ by $\theta$, then for small changes $\Delta \theta$, the criterion given by $\mathrm{Eq}(1)$ will be approximately proportional to

$$
\begin{aligned}
& \Delta \theta^{T}\left\{\int_{0}^{\frac{\pi}{\Delta T}} G_{20}^{\prime-1}\left(e^{j \omega \Delta T}\right) \times\right. \\
& \operatorname{Re}\left\{\left[\frac{\partial G_{1,0}^{\prime}\left(e^{j \omega \Delta T}\right)}{\partial \theta}\right]\left[\frac{\partial G_{1,0}^{\prime-1}\left(e^{-j \omega \Delta T}\right)}{\partial \theta}\right]^{T}\right\} \times \\
& \left.G_{2,0}^{\prime-1}\left(e^{-j \omega \Delta T}\right) d \xi(\omega)\right\} \Delta \theta
\end{aligned}
$$


Letting $\Delta T \rightarrow 0$, we obtain the optimization criterion for change detection in continuous-time models. It will be given by

$$
\begin{aligned}
& \Delta \theta^{T}\left\{\int_{0}^{\infty} G_{2,0}^{-1}(j \omega) \times\right. \\
& \operatorname{Re}\left\{\left[\frac{\partial G_{1,0}(j \omega)}{\partial \theta}\right]\left[\frac{\partial G_{1,0}(-j \omega)}{\partial \theta}\right]^{T}\right\} \times \\
& \left.G_{2,0}^{-1}(-j \omega) d \xi(\omega)\right\} \Delta \theta
\end{aligned}
$$

For easy reference, we denote $\mathrm{Eq}(4)$ or $\mathrm{Eq}(5)$ by $\Delta \theta^{T} M_{\theta} \Delta \theta$.

\section{Connection to Input Design for Parameter Estimation}

It can be shown [3] that under the assumptions of Theorem 1.1, the information matrix corresponding to $\mathcal{M}_{0}$ can be written as $M_{u}+M_{c}$ where $M_{c}$ is not input dependent and $M_{u}$ is proportional to $M_{\theta}$.

The information matrix asymptotically gives the covariance of any unbiased efficient estimator of $\theta$. Hence, maximizing a scalar function of the information matrix with respect to design variables is the topic of the statistical experiment design for precise estimation of parameters.

Now assume that only the changes in a subset of $\theta$ is of interest and should be monitored. We have the following theorem

Theorem 2.1 Assume that the parameter vector $\theta$ is partitioned into $\theta=\left(\theta_{1}^{T}, \theta_{2}^{T}\right)^{T}$, and $M_{\theta}$ is partitioned

$$
M_{\theta}=\left[\begin{array}{ll}
M_{11} & M_{12} \\
M_{12}^{T} & M_{22}
\end{array}\right]
$$

accordingly. It is desired to test the small changes in $\theta_{1}$. The minimum (worst) value of $\Delta \theta^{T} M_{\theta} \Delta \theta$ with respect to $\Delta \theta_{2}$ is assumed at $\Delta \theta_{2}=-M_{22}^{-1} M_{12}^{T} \Delta \theta_{1}$. This minimum value is

$$
\Delta \theta_{1}^{T}\left(M_{11}-M_{12} M_{22}^{-1} M_{12}^{T}\right) \Delta \theta_{1}
$$

Proof: The proof is straightforward and based on minimizing the Kullback information with respect to $\Delta \theta_{2}$. For easy reference, we denote $E q(\eta)$ by $\Delta \theta_{1}^{T} M_{\theta}^{\prime} \Delta \theta_{1}$.

Optimal input design is thus reduced to maximization of $\Delta \theta M_{\theta} \Delta \theta$ or $\Delta \theta_{1} M_{\theta}^{\prime} \Delta \theta_{1}$ subject to constraints. It requires a priori knowledge about the change $\Delta \theta$ or $\Delta \theta_{1}$. In absence of such prior information, one may for example maximize the determinant of $M_{\theta}$ or $M_{\theta}^{\prime}$. These choices of optimization criterion are respectively known as $D$ and $D_{s}$ optimality in the statistical experiment design literature [4].

\section{Optimal Inputs for Diagnosis}

Assume that the parameter of the model $\theta$ is a function of some physical parameter denoted by $\beta$, i.e. $\theta=F(\beta)$. It is of interest to monitor changes in $\beta$. Replacing $\Delta \theta \approx \frac{\partial F}{\partial \beta} \Delta \beta$, the optimization criterion is approximately given by $\Delta \beta^{T} M_{\beta} \Delta \beta$ where

$$
M_{\beta}=\left(\frac{\partial F}{\partial \beta}\right)^{T} M_{\theta}\left(\frac{\partial F}{\partial \beta}\right)
$$

Now, partition $\beta=\left(\beta_{1}^{T}, \beta_{2}^{T}\right)^{T}$ and assume that only the subset $\beta_{1}$ is of interest. Partition $M_{\beta}$ as in $\mathrm{Eq}(6)$ and obtain the criterion $\Delta \beta_{1}^{T} M_{\beta}^{\prime} \Delta \beta_{1}$ defined similar to $\operatorname{Eq}(7)$, cf [2].

\section{Conclusion}

In the paper, we have discussed the question of design of optimal input signals for detection and diagnosis. Our suggested design of inputs is based on full knowledge of the process in the normal operating conditions. However, no information about the possible faults is assumed given except for that they are small. The equivalence between optimal input design for fault detection and parameter estimation is established.

\section{References}

[1] R. H. Middleton and G. C. Goodwin. Digital Control and Estimation. Prentice Hall Information and System Science Series. Prentice Hall, 1990.

[2] P. Sadegh, H. Madsen, and J. Holst. Optimal input design for fault detection and diagnosis. Technical report, Institute of mathematical Modeling, Lyngby, Denmark, 1995.

[3] G. C. Goodwin and R. L. Payne. Dynamic System Identification: Experiment Design and Data Analysis. Academic Press, 1977.

[4] V. V. Fedorov. Theory of Optimal Experiments. Academic Press, 1972. 